\title{
The Fate of the Ultimobranchial Body of the Albino Rat with Special Reference to the Formation of the Thyroid Gland.
}

\author{
By \\ Shooichi Sugiyama. \\ From the Anatomical Institute, Nagoya Imperial University \\ (Director: Prof. Dr. Ch. Togari). \\ With five figures on Plate $\mathrm{X}$.

\section{Introduction.}

The question as to the relationship between the ultimobranchial body and the thyroid anlage has been repeatedly reviewed and discussed, yet no satisfactory answer has been obtained. Zuckerkandl (1903), Henneberg (1905), and Rogers (1926) have already described that the thyroid parenchyma in the rat is partly derived from the ultimobranchial body, but I have obtained other results than from these authors.

\section{Material and Methods.}

The embryos which I used for this study are the following: $6 \mathrm{~mm}$, $7 \mathrm{~mm}$., and romm. embryos in the greatest length; and also $12 \mathrm{~mm}$., $15 \mathrm{~mm}$., I $7 \mathrm{~mm}$., $18 \mathrm{~mm}$., $21 \mathrm{~mm}$., $22.5 \mathrm{~mm}$., $25 \mathrm{~mm}$., $30 \mathrm{~mm}$., and $40 \mathrm{~mm}$. embryos in the crown-rump length. The total number of embryos which were sacrificed for this study was 24. The fixing fluids employed were Zenker's, Helly's and Regaud's fluids. The materials were imbedded in celloidin-paraffin. The embryos were cut in transverse or sagittal sections of $s \mu$. or $10 \mu$. thick. Most of the sections were stained with hematoxylin and eosin, sometimes by Heidenhain's iron hematoxylin.

\section{Personal Observations.}

THE IOMM. STAGE

The ultimobranchial body is well separated for the first time from the 
pharyngeal pouch, and appears along the dorsomedial surface of the lateral lobe of the thyroid anlage. It is a somewhat irregular ellipsoid, in which a cavity exists having no contents (fig. I), and is bordered distinctly from the thyroid anlage by a thin layer of the mesenchyme surrounding the thyroid anlage. The cells of the ultimobranchial body are arranged very irregularly around the cavity, and their cell boundaries are indistinct. Their nuclei are mostly of one size, some are round, some short-oval, and few irregular-shaped. Its chromatin network is generally loose, but somewhat more dense than that of the nuclei of the cells of the thyroid anlage. A few nucleoli are contained in the nucleus. The cytoplasm is homogeneous, and stains faintly with eosin, and in general is small in amount. The mitoses in the body are seen in small numbers; on the other hand, the degenerating cells having metachromatic chromatins are considerably frequent.

\section{THE I $2 M M$. STAGE}

The ultimobranchial body is a long ellipsoid, and is almost in the same position as the one in the former stage. The cephalic part of the body is located nearer to the dorsomedial surface of the lateral lobe of the thyroid anlage. The cells of the ultimobranchial body are of the same general appearance as they were before (fig. 2). The cavity within the body is reduced in size, and becomes irregular in shape. Along the margin of the cavity, there are cell detrituses faintly stained with eosin. The cell arrangement in the body is very irregular, as before. The cytoplasm, which appears like a honey comb, is especially seen in the cell lining the cavity. The degenerating figures of the nuclei are more numerous than were found in the former stage (fig. 2), and occur as metachromatic chromatin blocks, or deeply stained nuclei provided with the obscure chromatin network, in both the central and peripheral part of the body.

\section{THE ISMM. STAGE}

The ultimobranchial body in this stage is smaller than in the prior stage, and is located nearer to the lateral lobe of the thyroid anlage. In some places of the caudal part, there are attachments between this body and the thyroid anlage, in other places a thin layer of the mesenchyme intervenes. As compared with the cells of the thyroid anlage, the cells of the body are more closely packed (fig. 3). The cavity within the body is reduced in size, and is irregular in shape; and along the margin of the cavity the cell detrituses stained with eosin are seen. Sometimes the detrituses of the cells are found within the cavity too (fig. 3). Degenerating figures of the cells are increased very conspicuously (fig. 3 ), and the mitoses are sometimes observed. 


\section{THE ${ }_{77}$ MM. STAGE}

The ultimobranchial body in this stage, being decreasde in size and irregular in shape, is included entirely within the expanding lateral lobe of the thyroid anlage (fig. 4). Most parts of the body directly touch the thyroid parenchyma. The cavity within the body is reduced in size, and contains cell detrituses. The cells of the ultimobranchial body are arranged irregularly and closely packed. The degenerating figures of the nuclei are often found, on the other hand, the mitoses are sometimes seen. Capillaries appear for the first time in the body, but are small in number.

\section{THE I 8MM. STAGE}

The ultimobranchial body is buried entirely in the lateral lobe, and is very irregular in shape, sending off its cell cords. These cell cords are sometimes so close to the thyroid parenchyma that the boundary between both is indistinguishable, sometimes are bordered from the thyroid parenchyma by the interstitial connective tissue. The cavity within the body becomes indistinct. The cells of the ultimobranchial body generally have the regularly round or oval nuclei, as compared with the ones of the cells of the thyroid anlage. Their nuclei are assembled together densely. The cytoplasm is inconstant in staining, sometimes stains deeply, and sometimes scarcely at all. The mitoses in the cells are seldom found, however the degenerating figures in them are often seen.

\section{THE 2I-22.5MM. STAGE}

The ultimobranchial body appears within the lateral lobe of the thyroid anlage still as an irregularly shaped structure having a small cavity. In the preparations which were fixed by Regaud's fluids and stained by Heidenhain's iron hematoxylin, the ultimobranchial body is colored darkly, and can be easily distinguished from the thyroid parenchyma. The cells of the body. are arranged as though they were stratified, and those directly lining the cavity are mostly clear, their nuclei are faintly stained and slightly irregular in shape, suggesting degenerative features, but a few mitoses are found.

\section{THE 25 MM. STAGE}

The ultimobranchial body generally cannot be seen within the expanding lateral lobe of the thyroid gland, but in certain cases the body is found as a small cell cord containing a tiny cavity. The cells directly lining the cavity are clear in general, and their nuclei are faintly stained and slightly irregular in shape. The thyroid follicles appear for the first time.

\section{THE $30 \mathrm{MM}$. STAGE}

The ultimobranchial body in general cannot be recognized within the 
lateral lobe, and appears rarely in the same manner as before.

THE 40 MM. STAGE

The ultimobranchial body can seldom be found within the lateral lobe in the same manner as before (fig. 5).

\section{Summary and Discussion.}

The ultimobranchial body appears in all vertebrates, from such lower forms as the fish and amphibia to higher ones as the mammalia and the human being. Besides the name, "ultimobranchial body", it is sometimes called : "lateral thyroid anlage" (Born), "postbranchial body" (Maurer), "supracardial body" (Van Bemmeln), and "telobranchial body" (Elze). There are many arguments as to the fate of this body. Especially in the mammalia and the human being, the ultimobranchial body has such an intimate relation with the thyroid anlage, so often called the median anlage, which arises from the pharyngeal floor, that naturally the questions arise among authors as to whether the ultimobranchial body takes a part in forming the thyroid gland or not.

Now in accordance with literature on the ultimobranchial body of man and mammalia: (A) Some persist in saying that it forms the thyroid gland itself. (B) Some say that it has no relation at all in forming the thyroid gland but degenerates or forms the cyst or "traubige Drüse". (C) Some recognize its close relation with the thyroid anlage, but still question its participation in the formation of the thyroid parenchyma.

Born (1883), Nicolas (1897), Tourneux and Verdun (1897), insisted on the (A) theory. Zuckerkandl (r903) stated that after the ultimobranchial body unites with the thyroid anlage in the ro- $x \mathrm{~mm}$. rat embryo, it forms the thyroid parenchyma. Henneberg (1905) also said that in the rat embryo, at the end of the 16 th day, the body fuses with the thyroid anlage, and forms a part of the thyroid gland.

Badertscher (1918) insisted that this.body in the pig absolutely participates in the formation of the thyroid gland, but the part formed from this body is comparatively small, and the cystoid follicles in the thyroid gland are derived from this body. Rogers (1926) also supported the theory, that the body forms the thyroid parenchyma in the rat. Weller (1933) wrote thus : In man, three primordia, one thyroid anlage and two ultimobranchial bodies fuse in the $13 \mathrm{~mm}$. stage into a single structure morphologically, which however is composed of two different types of tissue histologically. The thyroid anlage undergoes little change, until the ultimobranchial bodies become entirely similar to the thyroid anlage in histological appearance. Then the 
differentiation of the epithelium into thyroid follicles begins throughout the entire complex.

Soulié and Verdun (1897), Herrmann and Verdun(1899) supported the (B) theory. Stewart (1918) announced that the ultimobranchial body in the cat is believed to be an essentially regressive structure due to the evidence found in cellular disintegration, reticulation and cyst formation. Fox (1908), Anderson (1922), Fischel (1929), Bonnet-Peter (1929), Mason (1931), Takashima and Hara (1934), Selle (1935), and Kingsbury (1914, 1935) insisted that the body does not participate in the formation of the thyroid gland.

The following have insisted on the (C) theory. Grosschuff (1896) explained that the ultimobranchial body in the mammalia fuses with the thyroid anlage, but said nothing about the fate after the stage of fusion. Sudler (1902) did not say anything about the fate of the body in man, though he recognized the structural continuity between the body and the thyroid anlage. Tandler (1909) declared that it is not definite whether this body in man forms the gland or not. Moody (I9I0) stated that the thyroid anlage and the body in the pig embryo unite with each other and become a single structure in the $20 \mathrm{~mm}$. stage, but both tissues are distinctly distinguishable even in the $45 \mathrm{~mm}$. stage. Rabl (I9II, 1922) explained in the guinea-pig embryo, the body becomes imbedded within the lateral lobe, but he did not discuss as to whether this body atrophies later or finally is transformed into the thyroid parenchyma. Grosser (1910) agreed entirely with this opinion, in man. Nor ris (1916) supported that the body in the human embryo remains as a compact mass of the epithelium on the dorsomedial aspect of each lateral lobe, and also said it is uncertain whether this body atrophies later or is transformed into the thyroid parenchyma. Watzka (1933) explained in mammalia and man, that the body disappears within the gland or remains as a cyst, and questioned the participation of this body in forming the thyroid gland. Politzer and $\mathrm{Hann}$ (I935) declared that the body in man embeds itself into the thyroid gland, and never degenerates, but rather it is transformed into glandular cell cords. He mentioned that he cannot decide whether these cords have morphologically and functionally the same significances as the thyroid anlage or not. Further Politzer (1936). said that the body in man is included in the lateral wing of the thyroid anlage, and is transformed into cell cords, also that it can hardly be distinguished from the thyroid anlage in the $50 \mathrm{~mm}$. stage. $\mathrm{He}$ concluded that the body exhibits different types of development in various kinds of animals, so we should hesitate to judge the fate of the ultimobranchial body by the results derived from a few kinds of animals.

According to my study with the albino rat embryo, in the $10 \mathrm{~mm}$. stage the ultimobranchial body has lost its connection with the pharyngeal pouch, 
and is already situated on the dorsomedial side of the lateral lobe of the thyroid anlage (fig. I), then face to face with the lateral lobe bordered by a thin layer of the mesenchyme. On this point, I have the same results as Zuckerkandl, Henneberg, Rabl, and Stewart. Its shape is an ellipsoid, and the cavity is recognized in the central part of the body (fig. I). In the I $2 \mathrm{~mm}$. stage, the body approaches to the lateral lobe of the thyroid anlage very closely (fig. 2), and the cavity shrivels, in which the cellular detrituses are found. In the I $5 \mathrm{~mm}$. stage, this body shows a tendency to be surrounded by the lateral lobe (fig. 3), and shrinks in size to an irregular oval shape. The cellular detrituses in the cavity increase more and more (fig. 3). In the $17 \mathrm{~mm}$. stage, the body is imbedded in the lateral lobe entirely (fig. 4), and shrivels more, and becomes very irregular in shape. In the $18 \mathrm{~mm}$. stage, the body is transformed into an irregular-shaped structure and sometimes has a small cavity in it. The body remains until the $22.5 \mathrm{~mm}$. stage, but in general disappears after the $25 \mathrm{~mm}$. stage. Because of this fact the ultimobranchial body can be considered as a temporary structure which appears only in the early embryonic period, and that it does not participate in the formation of the thyroid parenchyma, but sometimes remains as a cyst-like structure in the stage in which the typical thyroid follicles are formed.

There are several arguments as to the character of the cell of the ultimobranchial body in comparison with the cell of the thyroid anlage. Fox (1908) wrote that in the pig embryo the minute structure of the body is easily distinguishable from that of the thyroid anlage by its lymphoid chatacter, and Grosser (1910) stated about man, ,Auf den ersten Blick erscheint der ultimobranchiale Körper als kleinzelliges, intensiv färbbares Gewebe." Anderson (1922) described that the cells of the body in the 20-50mm. calf embryos are quite different histologically from the cells of the thyroid anlage. The nuclei of the cells of the thyroid anlage are of varying sizes and shapes, containing numerous small bits of chromatin peripherally placed, but the nuclei of the cells of the body are smaller, rounder, more often of one size, and more closely packed. Rogers (1926) said of the rat embryo that the cells are morphologically indistinguishable from the thyroid parenchyma. Kingsbury (1935) stated about the calf embryo that in the uniting period the body retains a chromatic character of its nucleus and scanty cytoplasm. Politzer and Hann (1935) mentioned that in man the cell cords originating from the body stain more darkly than the cell cords from the thyroid anlage, and that both are distinguishable from each other even in the $40 \mathrm{~mm}$. stage.

With regard to the cells of the body in the embryo of the albino rat which I used, I found in the early developmental stage, that the nuclei of the body are not so varying in size as those of the cell of the thyroid anlage, and are 
regularly round or oval in shape (fig. 2). Irregularly shaped nuclei are present far less in the body than in the thyroid anlage. The chromatin network of the nucleus is weak and loose, and contains one or two nucleoli, contrary to the lymphoid character that Fox, Grosser and others declared (fig. 2). In the later developmental stage, most cells of the ultimobranchial body show a suggestion of degeneration, and are inconstant in staining, especially the cells directly lining the cavity are clear and stain scarcely at all (fig. 5). On the other hand, their nuclei become faintly stained and irregular in shape.

There are further arguments, for and against, the degenerative figures in the ultimobranchial body. Zuckerkandl, Henneberg, Rabl, and Crisan all denied its occurrence, while Stewart accepted the degenerative figures of the cell. Kingsbury and Mas on also found it in the body.

According to my results, this body already shows a tendency to degenerate in the romm. stage, and after this stage the cell degenerates very often, and the cavity is filled with cell detrituses (fig. 3). Some nuclei become very deeply stained, and some are also metachromatic. Such degenerative nuclei are also found in the $12 \mathrm{~mm}$. stage (fig. 2). In the $15-17 \mathrm{~mm}$. stage, they are increased (fig. 3). On the other hand, during these stages, $I$ also sometimes found the mitoses of the cells of the body. After the $25 \mathrm{~mm}$. stage, I recognized the ultimobranchial body consisting of degenerative cells which are very clear (fig. 5). In the normal development of the rat, I cannot support the facts that Zuckerk andl and others found. Simply speaking, with respect to both the mitoses and degenerative figures of the cells, it seems to me that the gradient of both has a certain relation with the duration of the remaining of the ultimobranchial body.

As mentioned above, there is much information concerning the ultimobranchial body, but in my study of the embryo of the albino rat, this body shows the degenerative process gradually in the case of normal development of the thyroid gland, and then disappears generally in the $25 \mathrm{~mm}$. stage, but sometimes remains as a small cell cord having a tiny cavity (fig. 5).

\section{Conclusion.}

The ultimobranchial body in the embryo of the albino rat is in general a temporary structure, which appears only in the early embryonic period, and disappears in the $25 \mathrm{~mm}$. stage in which typical thyroid follicles occur, that is, the ultimobranchial body does not participate in forming the thyroid parenchyma.

I wish to express my best thanks to Prof. Dr. Togari for his con- 
tinued guidance and criticism and to assistant Prof. Dr. Yamada for his helpful advice throughout the progress of this work.

\section{References.}

I) Anderson, E. L., (1922). Anat. Rec. 24, 25.

2) Badertscher, J. A., (1918). Amer. J. Anat. 23, 89.

3) Bonnet-Peter, (1929)- Lehrbuch der Entwicklungsgeschichte, 5 aufl.

4) Born, G., (1883). Arch. Mikr. Anat. 22, 271.

5) Crisan, C., (1935). Z. Anat. Entw. 104, 327.

6) Fischel, A., (1929). Lehrbuch der Entwicklung des Menschen.

7) Fox, H., (1908). Amer. J. Anat. 8, 187.

8) Grosser, O., (1910). Anat. Anz. 37, 337.

9) Grosschuff, K., (1896). Anat. Anz. I2, 497.

ro) Henneberg, B., (1905). Anat. Hefte. 28, 287.

II) Herrman and Verdun, (1899). (Cited from Zuckerkandl).

12) Kingsbury, B. F., (1914). Anat. Anz. 47, 609.

13) Kingsbury, B. F., (1935). Amer. J. Anat. 56, 445.

14) Kingsbury, B. F., (1935). Anat. Rec. 6r, I55.

I5) Mason, V. A., (193I). Amer. J. Anat. 49, 43.

16) Moody, R. M., (Igro). Anat. Rec. 4, 429.

17) Nicolas, (1897). Bibliogr. Anat. (Cited from Zuckerkandl).

18) Norris, E. H., (1916). Amer. J. Anat. 20, 41 I.

19) Politzer, G., (1936). Z. Anat. Entw. 105, 429.

20) Politzer and Hann, (1935). Z. Anat. Entw. 104, 670.

2r) Rabl, H., (I9ri). Verh. Anat. Ges. 25, 157.

22) Rab!, H., (1922). Arch. Mikr. Anat. 96, 210.

23) Rogers, W. M., (1926). Amer. J. Anat. 38, 349.

24) Selle, R. M. L., (1935). Amer. J. Anat. 56, 16r.

25) Stewart, F. W., (1918). Amer. J. Anat. 24, 191.

26) Soulié and Verdun, (1897). (Cited from Zuckerkandl).

27) Sudler, M. T., (rg02). Amer. J. Anat. I, 39r.

28) Takashima and Hara, (1934). Z. Anat. Entw. 102, 409.

29) Tandler, J., (1909). Anat. Hefte $38,393$.

30) Tourneux and Verdun, (1897). J. Anat. Phys. 23.

3I) Watzka, M., (1933). Z. Mikr. Anat. Forsch. 35, 485.

32) Weller, G. L., (1933). Contrib. Embryol. 141, 93.

33) Zuckerkandl, E., (1903). Anat. Hefte. 21, I.

\section{Explanation of the Plate.}

Fig. r. Photomicrograph of a transverse section of the ultimobranchial body in the $10 \mathrm{~mm}$. embryo. The ultimobranchial body appears along the dorsomedial side of the thyroid anlage, and the cavity is found within the body.

(Zenker. Hansen's hematoxylin and eosin.) $\times 85$. 
Fig. 2. Photomicrograph of a transverse section of the ultimobranchial body in the $12 \mathrm{~mm}$. embryo. The ultimobranchial body is near the lateral lobe. The nuclei of the cells of the body are mostly round or short-oval in shape, and the irregular-shaped nuclei are very few. Degenerative figures of the nuclei are found.

(Zenker. Hansen's hematoxylin and eosin.) $\times 350$.

Fig. 3. Photomicrograph of a sagittal section of the ultimobranchial body in the $15 \mathrm{~mm}$. embryo. The ultimobranchial body is surrounded partly by the expanding thyroid anlage. The degenerative figures of the nuclei in the body are found very often. The reduced cavity of the body is filled with cell detrituses.

(Zenker. Hansen's hematoxylin and eosin.) $\times 350$.

Fig. 4. Photomicrograph of a transverse section of the ultimobranchial body in the $17 \mathrm{~mm}$ embryo. The ultimobranchial body is entirely included within the lateral lobe of the thyroid anlage. The cells of the body are arranged irregularly, and closely packed.

(Zenker. Hansen's hematoxylin and eosin.) $\times 220$.

Fig. 5. Photomicrograph of a transverse section of the ultimobranchial body in the $40 \mathrm{~mm}$. embryo. The ultimobranchial body appears as a small cell cord containing a tiny cavity, and suggests a regressive structure.

(Zenker. Hansen's hematoxylin and eosin.) $\times 380$.

\section{Abbreviations.}

AN. Thyroid anlage.

C. Cavity of the ultimobranchial body.

D. Degenerative figure of the nucleus.

F. Follicle.

I.C.T. Interstitial connective tissue.

LI. Lateral lobe of the thyroid anlage.

TR. Trachea.

U. Ultimobranchial body. 
AN. TR.

D.

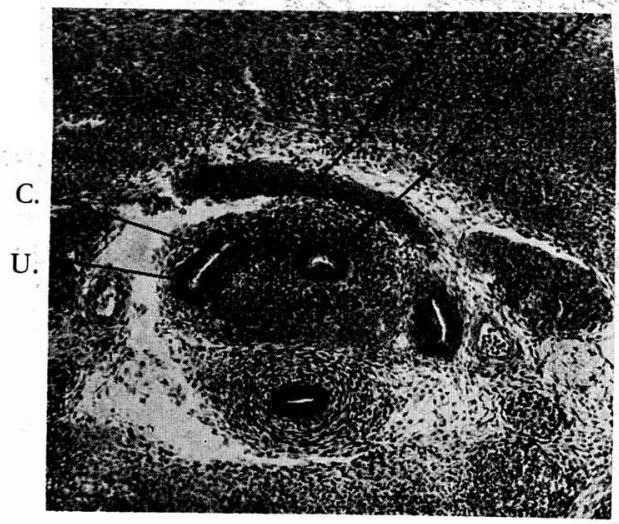

Fig. I.

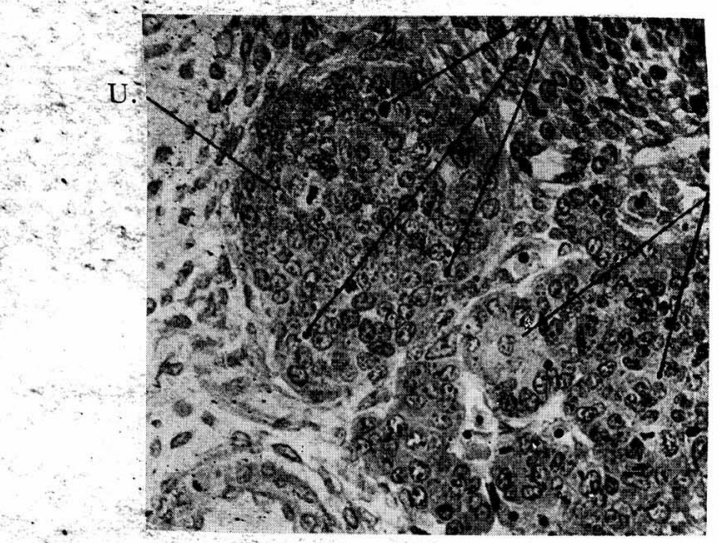

LL.

Fig. 2.

LL.

U.

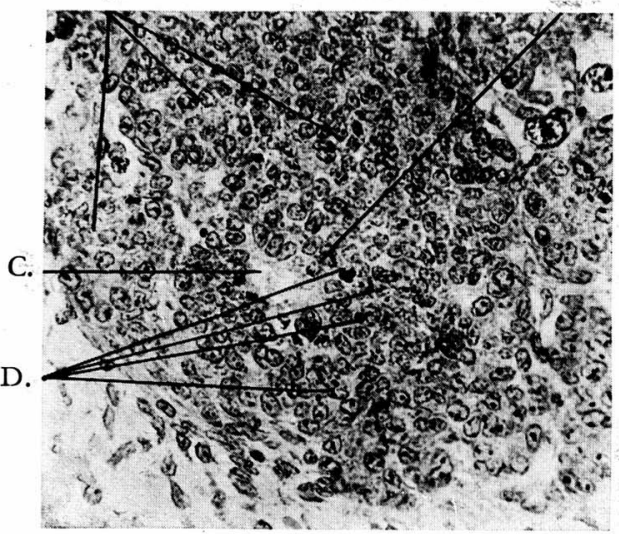

Fig. 3.
U.

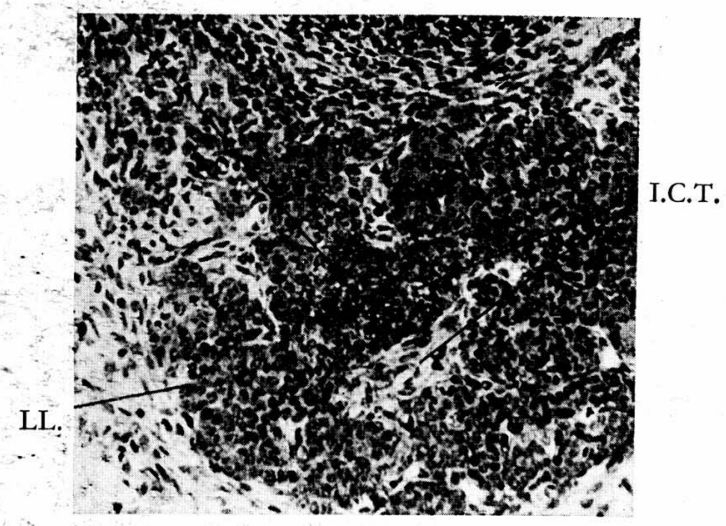

Fig. 4 .

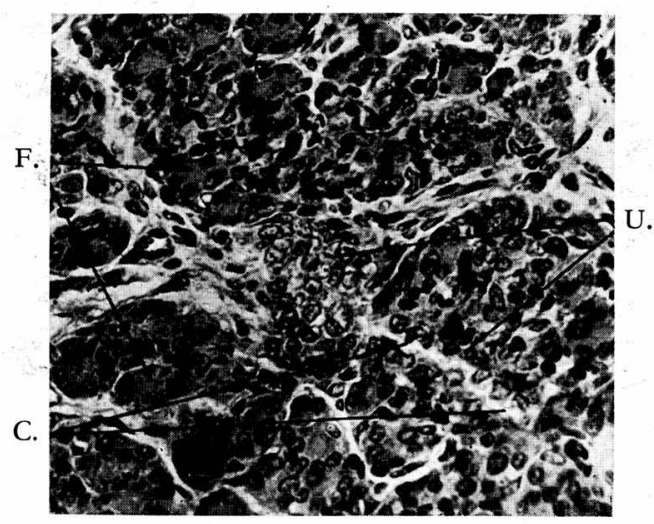

- Fig. 5. 